\title{
Learning Beyond the Basics of Cadaveric Dissection: a Qualitative Analysis of Non-academic Learning in Anatomy Education
}

\author{
Erin Parker $^{1}$ (D) Virginia Randall ${ }^{2}$ (10) \\ Accepted: 27 October 2020 / Published online: 10 November 2020 \\ (C) This is a U.S. government work and not under copyright protection in the U.S.; foreign copyright protection may apply 2020
}

\begin{abstract}
Rapidly advancing technologies have undeniably altered how medical education is taught; the anatomy lab is no exception. With a recent shift away from traditional cadaveric dissection and towards technology-based learning methods, medical educators are left wondering what students learn beyond the basics of anatomy during their course of human dissection. Especially considering the recent dramatic changes to the way medical education is conducted in the new era of a global pandemic, we must ensure students are not missing learning experiences that are critical to their development as future physicians. What are students gaining from cadaveric dissection that they otherwise could not gain from technology-based learning alone? Thematic qualitative analysis was used to review surveys collected from four anatomy classes over a two-year period; line-by-line coding of the surveys was then arranged into themes representative of specific learning experiences gained uniquely from human dissection. These themes demonstrated evidence of professional identity formation, self-reflection, and building teamwork skills; importantly, many students demonstrated a shift in thinking about their cadaver as less of a tool and more of a patient, which allowed them to generalize their experience in lab towards their future careers in medicine. These unique learning experiences cannot be replicated with technology-based learning alone. While cadaveric dissection may play a smaller role in the future of medical education, we must ensure we continue to provide students with learning experiences that are critical to their development as medical providers, especially if these experiences go beyond the anatomy basics.
\end{abstract}

Keywords Cadaver dissection · Qualitative analysis · Gross anatomy education · Medical education $\cdot$ Non-academic learning

\section{Background}

Advancements in technology over the last decade have left many questioning whether traditional cadaveric dissection is worth the time, effort, and cost it presents to medical schools. We use the umbrella term of technology-based learning methods to refer to any teaching methods based on virtual/ visualization technologies in place of traditional cadaveric dissection; we use the term distance-learning to refer to any form of anatomy education not taking place physically at the student's learning institution. Previous research has suggested

Erin Parker

1 School of Medicine Class of 2021, Uniformed Services University of the Health Sciences, Bethesda, MD, USA

2 Associate Professor of Pediatrics, Uniformed Services University of the Health Sciences, Bethesda, MD, USA that when student learning is based primarily on the outcome of exam performance, students using three-dimensional visualization technologies appear to perform equivalently to their student counterparts who use traditional cadaveric dissection [1]. This is not to imply that these two teaching paradigms are equivalent in all aspects, but it does suggest that technologybased learning may be equivalent to cadaveric dissection if exam performance is the primary outcome. If basic anatomical knowledge is learned without the use of human cadavers, is there a reason to continue traditional dissection?

Studies on medical students' reactions to the use of cadaveric dissection in anatomy education have been previously reported. In one study in a German medical school, the authors analyzed data using Likert scales and found that students responded favorably overall to the dissection process due to its ability to teach professionalism, teamwork, and coping strategies [2]. Responses from physicians regarding the impact cadaveric dissection had on their futures as professionals in medicine have been previously assessed, finding that the hands-on experience of the gross anatomy lab as students 
provided motivation for many physicians to pursue surgical specialties [3, 4]. Our present study builds on previous research, but we have sought to gain a more diverse subset of data by distributing an anonymous open answer formatted survey to allow the broadest range of responses possible; we are uniquely focused on looking from the medical student perspective with regard to dissection. We have sought to further describe the reactions these students have as they begin the process of human dissection and how these reactions shape their outlook as future physicians.

Our study being reported here is based on responses from multiple classes of students from the Uniformed Services University of the Health Sciences (USUHS), the military's medical school; we thus draw data from groups of students who are developing skills not only as future physicians but also as military officers. This presents a unique background for our data as students already have an emphasis on leadership training prior to their experiences in the anatomy lab [5]. Despite collecting data from this population, we feel our results are generalizable to larger populations as we have focused on many aspects of what is learned from human dissection and not just on leadership skills.

Given the comparable exam performance between students using traditional cadaveric dissection and students using technology-based learning methods, we initially set out to determine what it is that students learn from dissection that they would not otherwise gain from using technology-based learning alone. This goal gained a unique context amid the 2020 COVID-19 pandemic, in which medical schools have been forced to make drastic changes to their normal curriculum to comply with social distancing standards.

Despite a goal of determining what learning experiences occur in the course of traditional human dissection, we are not arguing against the use of newer technology-based learning methods. Indeed, technological advancements cannot be denied a place in medical education; ignoring the benefits of new educational techniques is a dangerous pitfall. We must remain objective when considering new teaching methods, and not prefer one method over another simply for the sake of familiarity [6].

\section{Research Question}

To understand medical students' perceptions of anatomy education and non-academic learning in cadaveric dissection.

\section{Methods}

With Institutional Review Board approval, we used thematic qualitative analysis to study 197 surveys from four anatomy classes: two classes each year in 2018 and 2019 consisting of first-year students completing musculoskeletal dissection and second-year students completing their neuroanatomy module. There were 695 total students in the four sessions of anatomy resulting in a return rate of $28 \%$. We emailed SurveyMonkey $\odot$ questionnaires to the students in the anatomy classes, who were assured of anonymity. No demographic data was collected. The questions were open ended:

Q1. What was your initial reaction when you walked into the cadaver laboratory on day one?

Q2. How has that changed?

Q3. What have you learned about the practice of medicine through your experience of dissection?

Q4. What have you learned about yourself through your experiences in the anatomy laboratory?

Q5. Do you have any other comments, questions, or concerns?

These questions were developed by building on $10+$ years of experience developing surveys for medical students. The theoretical construct behind these questions was evaluating for the presence of "threshold concepts," or non-factual learning points often won through emotional difficulty, that produce attitudinal/belief changes that are representative of ontological and transformative experiences [7,8].

Two researchers independently used line-by-line coding with constant comparison to code each of the four sets of surveys. Each set of codes were discussed until consensus. Similarly, each researcher independently arranged codes into themes which were discussed until consensus, forming thematic networks through analysis of the available data [9]. We used caution to keep to the exact words of the students and incorporate them into quotations illustrating themes.

The two researchers are at different stages in their careers and a generation (or two) apart, lending the advantage of differing viewpoints and interpretations of the student responses.

The surveys and thematic analysis were all completed before the COVID-19 pandemic began in the USA; after the start of the pandemic, there was a necessary re-thinking of how to teach anatomy in our school, which heretofore had been cadaveric based.

\section{Results}

The following themes were developed after analyzing the available data and identifying patterns within student's responses. They have not been organized in a hierarchical order; rather, we feel that each theme represents a specific learning experience that is equally important to student development and is a direct result of their interaction with human cadavers. Figure 1 below is a concept map developed by the authors to 


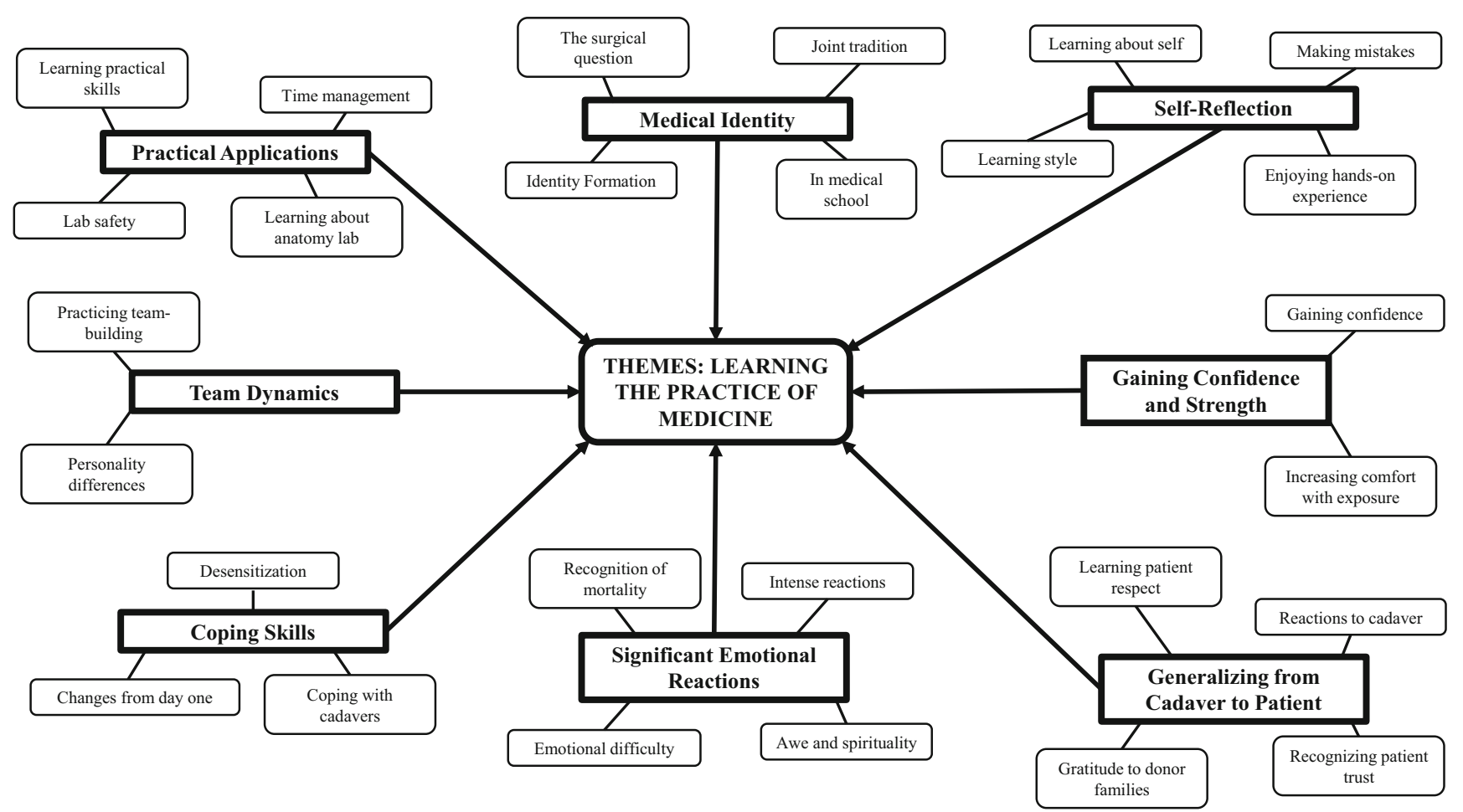

Fig. 1 Concept map displaying network of themes discovered through analysis of student responses

display the themes discovered through analysis of student responses.

\section{Medical Identity}

The theme of medical identity encompasses the ideas students develop about themselves as future medical professionals through the practice of cadaveric dissection. This includes students' recognition of beginning the medical school experience ("lab made me realize that I'm really studying medicine and in medical school") as well as the realization of taking their own place in the long line of medical professionals who came before them ("I feel a connection with the roots of the profession”). This theme includes students' progression towards identity formation as a result of their experiences working hands-on with cadavers ("I feel my identity as a future physician has been developed by anatomy lab"), as well as the beginnings of working out prospective specialties they may choose to pursue in the future - whether it be a positive or negative reaction to surgery ("lab class makes me really like surgery due to the comfort level I have"; "standing over a table all day is probably not going to be in my future").

\section{Self-Reflection}

Self-reflection is a broad theme meant to describe aspects that students learned about themselves through their exposure to anatomy lab. Notably, this includes what students found out about their own learning style ("I learned how much of a practical learner I am"; "I learn better when I'm teaching things to my group"), their opinions on hands-on experience ("I really enjoy a hands-on approach to medicine"; "dissection allows me to better understand spatial relationships"), and what they feel they have learned about themselves through the experience of cadaveric dissection ("I've identified personal strengths and weaknesses when working with a cadaver"; "I've learned I need to work on my patience"). Finally, this theme explores students' reactions to making mistakes and learning to manage expectations ("anatomy lab has helped me see that doing is learning, and that it doesn't have to be perfect to learn").

\section{Coping Skills}

Given the disconcerting nature of dealing with cadavers, this theme describes students' mechanisms for coping with the sometimes unsettling reality of working with a deceased individual. This includes generalized coping skills ("I felt a little better once we flipped the cadaver over") as well as students' descriptions of feeling desensitized to the cadavers after a period of time ("I feel like I've dissociated now, I have trouble reminding myself that I'm working with a deceased individual"; "the cadaver slowly became less real to me."). This theme covers the changes students underwent as a result of working in the lab over time, and how these changes helped them cope with the reality of what they were experiencing ("with many sessions the uneasiness went away and I became much more 
comfortable"; "once we started dissecting I became much more comfortable around cadavers").

\section{Gaining Confidence and Strength}

As students progressed through their initial reactions to the cadaver lab, many expressed a new-found confidence in themselves as a result of being capable of completing cadaveric dissection. Thus, this theme describes student's reactions to gaining confidence/strength through being successful at dissection ("I learned medical school is hard, but I can do it"; "I feel stronger for having done something successfully that I always feared"; "I thought I could never work on a cadaver, because the idea scared me. Now I feel confident in my abilities"). For students, this newly built confidence was the result of accomplishing something that had once provoked anxiety — working with a cadaver.

\section{Significant Emotional Reactions}

Working with cadavers may understandably provoke emotional and visceral reactions for some students; this theme describes these reactions. This includes the concept of emotional support and difficulty students may face ("I felt very unsettled leaving lab"; "I felt really lonely and sad"; "certain labs were difficult for me emotionally") as well as intense reactions which some students reported ("I had a very visceral reaction and it took me a few minutes to calm down"; "when I finally saw our cadaver, it was nearly an out-of-body experience"). This theme includes students' responses that described a feeling of awe and spirituality when interacting with their cadaver ("the order of the body is incredible, and I feel this is something that has convinced me there is a God"; "I felt the instant need to be reverent"; "there was a feeling of awe"). Finally, this also includes student's reactions to their own sense of mortality as a result of working with cadavers ("cadaver lab brought my attention to my own mortality").

\section{Generalizing from Cadaver to Patient}

One key aspect students perceived during their time in lab was the learned skill of generalizing what they were doing with their cadaver to what they will one day be doing with patients. This theme demonstrates this skill with concepts such as students learning patient respect through cadaveric dissection ("I've learned to respect each and every patient, dead or alive"; "I've learned that being a successful physician means being attune to your patients in every single way, dead or alive") as well as students recognizing the gravity of patient trust ("I'm overwhelmed by the trust the donors have in us"; "I've learned to value and appreciate that patient's trust us"). This also includes the reactions students had to their cadavers ("the face gets me every time"; "occasionally seeing an undissected part will be unsettling") and the gratitude they feel towards the donors and the donor families ("I felt incredibly grateful to have the generosity of our cadaver's donation to my education"; "I am very grateful for the sacrifice the donor families made").

\section{Practical Applications}

Throughout the course of anatomy lab, students begin to have an appreciation for more of the "practical" learning that they gain as a result of working in a laboratory setting; this includes the idea of learning technical skills ("I'm getting more comfortable and have better control with a scalpel"; "I'm learning surgical skills"; "our skills have gotten dramatically better as lab has gone on") as well as time management ("going too slow you may not finish"). Some students also expressed a recognition of learning safety in the lab setting ("I learned to be safe with sharp tools"; "my team developed safety words when we move scalpels"). In addition, this theme also includes elements that students learned from spending time in an anatomy lab setting ("formaldehyde I think irritates my nose and lab gets cold"; "if your cadaver is obese the anatomy is much harder to find").

\section{Team Dynamics}

This theme includes a key aspect of medical student development: learning team building skills and how to work as a member of a cohesive unit. This includes the concept of team dynamics while dissecting ("I learned how to work with my peers"; "I learned I can work well in a team"; "the interpersonal dynamics of the dissection lab group have an impact on the learning experience for individuals"; "I'm learning to be a better team player"; "I've learned the importance of being a team player on a medical team").

\section{Learning Medicine beyond Anatomy Basics}

Students learn basic anatomy as a result of their time in lab; however, we found that students consistently commented on the things they learned about medicine beyond these anatomy basics. This theme includes what students learned about the art of medicine ("medicine is not just science, but an art"; "pride has no place in the proper care of patients"; "what just the body can tell you is incredible"), as well as what they learned about the significance of anatomic variation ("not everything looks like the textbook or is where the textbook thinks it should be"; "each patient is unique"; "variance is difficult to learn from textbooks, and crucial to practice"). This also includes how students correlated anatomy to other courses and to their learning of physical exam maneuvers ("I learned developmental correlates"; "anatomy influences body pathology" "I can correlate to the physical exam because I know what is going on underneath the skin"). Finally, this 
also included the student's reactions to their interactions with the faculty as well as their gratitude to the staff and teaching assistant facilitators.

\section{Discussion}

Cadaveric dissection allows students to form ideas of who they will become as physicians, who they are as individuals, how they interact within a team, and how they plan to treat patients in the future. The experience of cadaveric dissection facilitates student's development of coping skills and promotes gaining strength/confidence in their own abilities as future physicians. We propose that these learning experiences are equally as important as learning basic anatomical knowledge, and that these unique learning opportunities cannot be replicated through technology-based learning methods alone. The themes discovered through analysis of student reactions are evidence that students are learning principles of the practice of medicine beyond the basics of anatomy through their experiences with cadavers; some of these learning experiences are transformative: they result in students redefining themselves as future medical professionals.

This discussion takes on a unique urgency amid the global COVID-19 pandemic, in which dramatic changes to medical education have resulted in most students learning anatomy outside of the cadaver lab. Shifts in medical education towards a more technology-based curriculum were taking place prior to the start of COVID-19 and will undoubtedly continue after; the difference we must now face is the amount of choice medical educators have. Prior to the pandemic, medical educators had free choice in how they shaped their curriculum; given the new norms of social-distancing requirements, much of that choice has now been taken away. Our focus, therefore, should ultimately be on providing the highest standard of medical education while attempting to provide these crucial learning experiences despite the constraints of social-distancing and distance-learning.

Through our own exploration of the results developed from our study, we propose to separate the themes into three categories for better utilization: (1) themes that are unique to cadaveric dissection and would be difficult to replicate without cadavers; (2) themes that are associated with cadaveric dissection but could possibly be replicated without cadavers; (3) themes that are associated with cadaveric dissection but could easily be replicated without cadavers. Figure 2 lays out these categories in further detail.

Below is a description of some of the concepts learned in the cadaver laboratory that will be missing from an all-virtual platform; we make some suggestions as to how to provide medical students with an experience that will allow for similar learning. These suggestions are organized from easily replicable themes to less easily replicable themes as described in Fig. 2 above.
Theme: Practical Applications Gaining experience and practicing technical skills, especially for students who are considering surgery, is an important experience. We considered that this experience could be fostered by encouraging students to join their school's Surgery Interest Group or setting up shadowing opportunities for students interested in surgery at their home institution or regional hospital.

Potentially budding surgeons are not the only students, however, who benefit from the practical applications of anatomy lab; many students remarked on how they were able to better understand physical exam maneuvers after dissecting and viewing pro-sections of the various human joints in anatomy lab. This could be replicated by providing physical examination instructors with high-quality joint models for the students to use as they learn examination skills. Videos of arthroscopic surgery could also be a potential asset to young learners first being exposed to physical exam maneuvers.

Theme: Team Dynamics Team building was likewise an important skill students felt they learned around the dissection table; learning to manage team dynamics is a crucial skill for medical students to develop as they will one day be challenged to play different roles within a medical team caring for patients. Our study may differ from other medical schools regarding this experience as our work took place at the USUHS, the military's medical school; this institution dedicates a substantial portion of the curriculum to team building and leadership exercises throughout the 4 years of medical school, and therefore our sample population of students has additional background education in aspects of team dynamics and leadership.

Despite the added hours of team building/leadership education they receive, students at our institution still felt they gained added knowledge regarding team dynamics from their experience in lab. At our military institution, we recommended alerting the department responsible for our longitudinal leadership education course that a central team building experience may go missing for students if cadaveric dissection were to be replaced; this allows the department to augment their curriculum accordingly. At non-military institutions, it may be reasonable to adjust the curriculum to add in more team building exercises to continue to expose students to the challenges of working within different team dynamics.

Theme: Coping Skills Several students commented on their growth over the course of the anatomy lab curriculum, developing coping strategies to deal with the sometimes unsettling nature of working with cadavers. This is obviously difficult to replicate outside of a cadaver lab; however, we considered preparing a document to be distributed to students that describes how bodies are prepared following death, allowing an opportunity for students to prepare themselves if they experienced anxiety at the concept of death. 


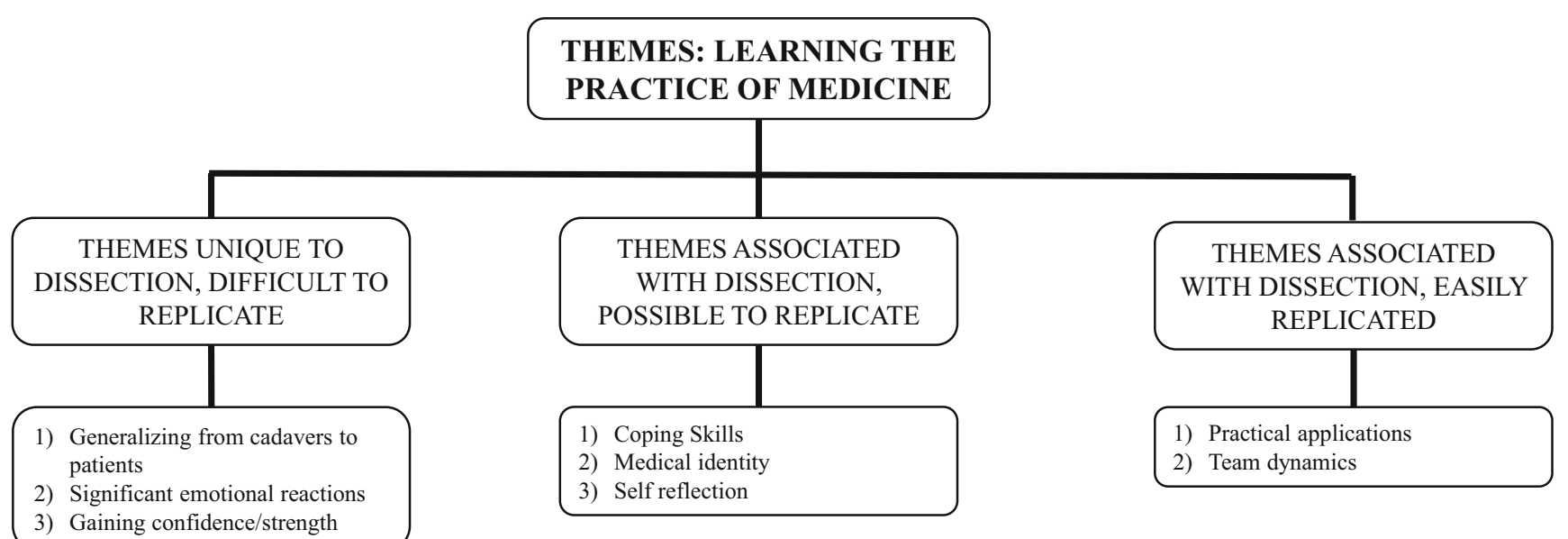

Fig. 2 Themes distributed into categories according to replicability outside of cadaveric dissection

Theme: Medical Identity Participation in cadaveric dissection helped some students realize their place in the long line of physicians/anatomists who came before them; we considered this to be a recognition of the joint tradition of medicine. Cadaveric dissection became a juncture for many students in which they realized they had entered the medical field and simultaneously became a part of something bigger than themselves. While this is also a difficult experience to replicate, we considered presenting students with a photo journal of past physicians such as da Vinci and Galen; this journal could also include pictures and brief biographical sketches of their current faculty.

Theme: Self-Reflection Encouraging self-reflection in our students is a necessary part of medical education and a crucial skill for future medical providers to develop; this is oftentimes a difficult concept to practice in a virtual learning world, and especially difficult as medical education shifts towards a focus of teaching to multiple-choice exams, which do not necessarily require integration/perspective of concepts in order to pass [10]. If cadaveric dissection (an experience which facilitates selfreflection within students) were to take a lesser role in our curriculum, we have considered placing additional emphasis within our first- and second-years' Humanities course on questions that encourage students to self-reflect on their futures as medical providers. Questions such as "what are your fears about becoming a physician?" may help students to begin to reflect on who they are as future medical practitioners; this would require further facilitated discussion in small groups.

\section{Limitations/Strengths}

This study was conducted in a military medical school in the US. Results may not be generalizable to other medical schools.

Our analysis of the above data took place in March of 2020 at the beginning of the COVID-19 pandemic; as such, the impacts distance-learning may have on anatomy education was not directly studied. This would make for an excellent future study to address the impacts of the COVID-19 pandemic on anatomy education.

A statistical analysis of how closely the reviewers agreed was not performed, and therefore an inter-rater reliability score is not available.

We are uniquely considering the reaction to dissection from the medical student perspective, with a larger sample size of 197 student responses.

\section{Conclusions}

With a shift towards distance-learning and increasing use of technology-based learning approaches, the lack of exposure to cadaveric dissection results in medical students missing out on crucial learning experiences that shape who they are as future medical providers. The experience of dissection provides an environment in which students are challenged to integrate concepts in order to solidify learned information - an experience that is already minimized in the modern age of teaching to multiple-choice exams. As a result of dissection, students are not only challenged to form their own identity within the medical field, but are also encouraged to self-reflect, build teamwork skills, and develop coping strategies for emotionally challenging experiences. Importantly, many students demonstrated a shift in thinking about their cadaver as less of a tool for learning and more as a patient; this shift encouraged students to begin generalizing their experiences in the cadaver lab towards their future experiences with patients. These unique experiences cannot be replicated with technologybased learning alone.

This is not to argue that there is no place for technologybased learning in the future of medical education; to avoid utilizing the benefits of new educational technologies is a dangerous pitfall. It is crucial to remain objective when 
considering new educational techniques, and not prefer one learning method simply for the sake of familiarity. We instead argue that if technology-based learning is to play a larger role in the future of medical education, we must ensure methods are in place to continue to provide students with the learning experiences that are critical to their development as future physicians. Future work, therefore, could focus on analyzing how these learning experiences could be replicated with little or no cadaveric dissection. We feel that research into the benefits/challenges of virtual platforms for anatomy education must involve medical students in the analysis and in proposing alternate activities. In addition, future research is needed to solidify how traditional dissection and modern technologybased learning can be integrated to produce a learning environment that is most beneficial to the success of future medical students. This is our fundamental duty as medical educators: providing the best foundation possible for the success of our students.

\section{Take Home Points}

- There are learning experiences unique to dissection that are equally as important to student development as learning basic anatomy. These experiences cannot be recreated with technology-based learning alone.

- The learning experiences gained through dissection help to foster the identity formation of students as they develop self-confidence and begin to define themselves as future medical providers, taking their place in the line of physicians who have come before them.

- The experience of cadaveric dissection provides an environment in which students learn integration of concepts and perspective as future medical providers; this is not otherwise readily available when there is only a focus on teaching to multiple-choice exams.

- During the distance-learning era of COVID-19 and beyond, it is essential that we ensure not only that our medical students pass their exams but that they are exposed to experiences critical to their development as future physicians.

Author Contributions All authors contributed to the study described herein. Material preparation and data collection was performed by Dr. Virginia Randall; analysis of data was performed by Dr. Virginia Randall and Erin Parker. The first draft of the manuscript was written by Erin Parker and both authors commented on previous versions of the manuscript. Both authors read and approved the final manuscript.
Funding This study took place at the Uniformed Services University and did not require funding to be completed. The authors have no financial interests related to this article.

Data Availability Not applicable.

\section{Compliance with Ethical Standards}

Disclaimer The opinions expressed are those of the authors and do not necessarily represent the position of the DoD or USU.

Conflict of Interest The authors declare that they have no conflict of interest.

Code Availability Not applicable.

\section{References}

1. Yammine K, Violato C. A meta-analysis of the educational effectiveness of three-dimensional visualization technologies in teaching anatomy. Anat Sci Educ. 2015;8:525-38.

2. Blockers A, Jerge-Bretzke L, Lamp C, Brinkmann A, Traue HC, Bockers TM. The gross anatomy course: an analysis of its importance. Anat Sci Educ. 2010;3:3-11.

3. Khan AN, Baig S, Zain S. Importance of cadaveric dissection in learning gross anatomy. Pakistan Journal of Medicine and Dentistry. 2014;3:31-5.

4. Ghazanfar, H., Rashid, S., Hussain, A., Ghazanfar, M., Ghazanfar, A., \& Javaid, A. (2018). Cadaveric dissection a thing of the past? The insight of consultants, fellows, and residents. Cureus URL: https://www.cureus.com/articles/11748-cadaveric-dissection-athing-of-the-past-the-insight-of-consultants-fellows-and-residents [accessed: 10 April 2020].

5. Will, J. S., \& Malave, B. (2019). Military physicians are not just physicians in the military: using leadership training in military graduate medical education to assure mission success. Journal of Military Medicine 1-3.

6. Norman G. The once and future myths of medical education. J Grad Med Educ. 2020;12:125-30.

7. Randall, V., Brooks, R., Montgomery, A., \& McNally, L. (2018). Threshold concepts in Medical Education. MedEdPublish; https:// doi.org/10.15694/mep.2018.0000176.1

8. Land R, Meyer J h, Smith J. Threshold concepts within the disciplines. Rotterdam: Sense Publishers; 2008.

9. Braun V, Clarke V. Using thematic analysis in psychology. Qual Res Psychol. 2008;3:77-101.

10. Cahill D, Leonard R, Weiglein A, von Ludinghausen M. Viewpoint: unrecognized values of dissection considered. Surg Radiol Anat. 2002;24:137-9.

Publisher's Note Springer Nature remains neutral with regard to jurisdictional claims in published maps and institutional affiliations. 